\title{
COMPARISON OF OPTIONS FOR BUILDING AN EMERGENCY CALL SYSTEM
}

\author{
Mohammed Omar Ahmed Abdulvasea, \\ TaizUniversity, Taiz, Yemen, \\ omaralmu2012@mail.ru
}

DOI: 10.36724/2664-066X-2020-6-I-16-22

\begin{abstract}
As the coronavirus pandemic has shown, it is extremely important to create special services for operational assistance to the population. The rapid, uncontrolled growth of emergency call traffic determines the relevance of resource pooling emergency call centers within the framework of System 112(system 112 is fully used in the territory of the Russian Federation, an analogue of this system is 911 in the USA). The effective use of the combined resource of operators in an emergency is an urgent scientific task. The article discusses the organization of the reception of emergency calls from the population and the distribution of applications between specific duty dispatch services.

We study the following approaches to the organization of emergency services:

Redirecting excess traffic to a common rapid response center;

Implementation of a distributed response center;

Formation of an operational reserve of operators in service areas.

The stages and the main functionality of the method of combining and efficient use of resources of the emergency call service system are determined in order to minimize the loss of operational information.
\end{abstract}

KEYWORDS: Emergency, emergency services, interactive voice menu, call centers, operational reserve.

\section{INTRODUCTION}

We will consider the possibility of using an additional resource of emergency response service center (ERSC) to service emergency calls in an emergency. Part of the calls from call centers in the emergency zone can be routed to the emergency response center. The prospects and the possibility of using emergency response service centers are reflected in a number of documents of ITU-T and the government of the Russian Federation [1, 2, 3]. The purpose of organizing such centers is to ensure the receipt of emergency calls from the population with minimal failures, as well as the preliminary processing of emergency information by operators. Under failure understood the direction of the call to the IVR system (IVR - interactive Voice Response) due to the busyness of all operators at the time of the emergency call. Such calls may be served with a delay. The speed of obtaining information from the emergency zone is reduced.

When developing a mathematical model and research the characteristic of complex communication systems in the theory of teletraffic, an approach is often used that offers to divide the system into separate modules. Imagine the system under study as a combination of several separate modules. This is a decomposition method $[4,5]$. 
We denote the total number of call centers in the system by the value $\mathrm{W}, \mathrm{j}=1.2 \ldots \mathrm{W}$ and the unified duty dispatch service by the UDDS (see Fig. 1). A dedicated rapid response center is an additional resource.For calculations, the RDA method was used. The name of the RDA method is formed by the system of indicators used: the value of $R$ indicates the average value of the excess load; D is its scattering(i.e., the difference between the variance and the average value); $\mathrm{A}$ - load intensity.

A special program is developed to calculate the characteristics of the system according to the method RDA. Table 1 presents the results of calculating the required number of operator jobs V_ERSC,received for the given requirements for call losses, namely for P_ERSC $\leq 0.001$ and $P \_E R S C \leq 0.01$. It was assumed that the average traffic level in the districts is at 10 Earl. In fact, the case of eight-fold growth in growth traffic in the emergency area is considered.

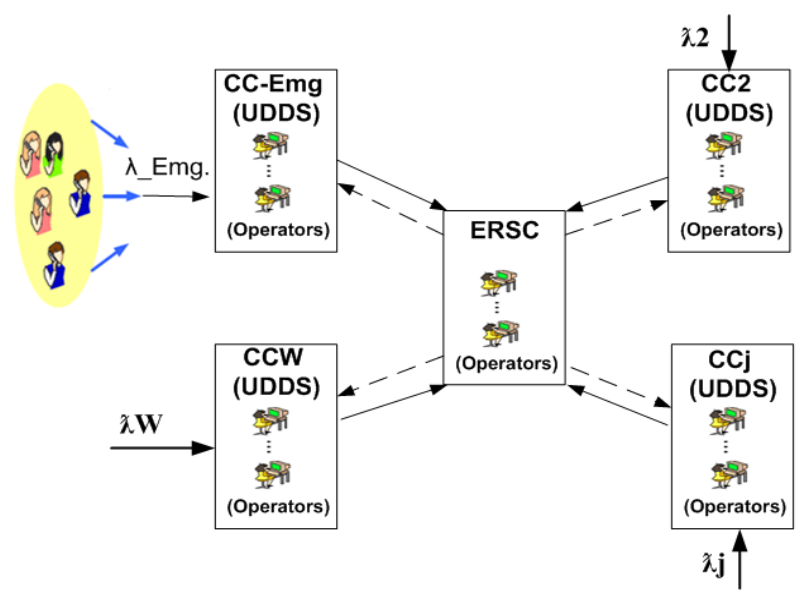

Figure 1. Variant of the implementation of the emergency response service center (solid lines - the path of service requests, dashed lines - the return path of processed electronic requests)

Table 1

The results of the calculation of the required number of workplaces of operators in the call center ERSC in case of emergency in one area (CC-Emg)

\begin{tabular}{|l|l|l|l|l|l|l|}
\hline \multicolumn{7}{|c|}{ Number of operatorjobs in CC-Emg (V_Emg) $=20$} \\
\hline $\begin{array}{l}\text { Incoming } \\
\text { load in } \\
\text { CC-Emg, } \\
\text { AEmg, Erl }\end{array}$ & $\begin{array}{l}\text { Total } \\
\text { excess } \\
\text { load, R }\end{array}$ & $\begin{array}{l}\text { Total } \\
\text { dispersion } \\
\text { of excess } \\
\text { load, D }\end{array}$ & Aeq & Neq & $\begin{array}{l}\text { Vreq } \\
\text { ERSC } \\
\text { when } \\
\text { PERSC } \\
\leq 0,001\end{array}$ & $\begin{array}{l}\text { Vreq_ } \\
\text { ERSC } \\
\text { when } \\
\text { PERSC } \\
\leq 0,01\end{array}$ \\
\hline 20 & 3,178 & 5.114 & 20 & 20 & 18 & 14 \\
\hline 30 & 11,402 & 12,363 & 30 & 20 & 29 & 24 \\
\hline 40 & 20,852 & 15.488 & 40 & 20 & 41 & 35 \\
\hline 50 & 30,605 & 16,927 & 50 & 20 & 53 & 46 \\
\hline 60 & 40,467 & 17.708 & 60 & 20 & 64 & 56 \\
\hline 80 & 60,320 & 18.505 & 80 & 20 & 87 & 78 \\
\hline
\end{tabular}

Table 2 presents the results of calculating the required number of workplaces of operators $\mathrm{V}$ (reqERSC) obtained for $\mathrm{P}$ ERSC $\leq 0.01$ in conditions when there is a sharp increase in emergency traffic in two regions.
Table 2

The results of the calculation of the required number of workplaces of operators in call center ERSC in case of emergencies in two regions when $\mathrm{P}$ ERSC $\leq 0.01$

\begin{tabular}{|l|l|l|l|l|l|}
\hline \multicolumn{6}{|c|}{ Number of operator jobs in CC (Vj) $=$ V_Emg $=20$} \\
\hline $\begin{array}{l}\text { Incoming } \\
\text { load in } \\
\text { CC-Emg, } \\
\text { AEmg, Erl }\end{array}$ & $\begin{array}{l}\text { Total } \\
\text { Excess } \\
\text { load, R }\end{array}$ & $\begin{array}{l}\text { Total } \\
\text { dispersion } \\
\text { of excess } \\
\text { load, D }\end{array}$ & Aeq & Neq & $\begin{array}{l}\text { Vreq_ERSC } \\
\text { PERSC } \leq 0,01\end{array}$ \\
\hline $20+20$ & 6.36 & 10.23 & 28.76 & 25 & 19 \\
\hline $30+30$ & 22.80 & 24.72 & 52.59 & 31 & 39 \\
\hline $40+40$ & 41.70 & 30.98 & 76.89 & 36 & 60 \\
\hline
\end{tabular}

Analyzing the obtained results, we can draw a preliminary conclusion that the organization of emergency response service center allows efficient service of excess traffic from several call centers (CC). For example, with a four-fold increase in traffic in each of the two districts of the emergency, it will be necessary to employ a significantly smaller number of jobs in the call center ERSC, all other things being equal (up to 60 jobs instead of 78 with eight-fold increase in traffic in the only emergency area).

We list the negative aspects of organizing a call center ERSC:

In irrational use of the resources of ERSC (only during emergency situations);

The throughput of the entire system can increase due to the organization of additional operator jobs in ERSC. At the same time, the positive aspects are:

The possibility of operational mutual assistance to several call centers at the same time.

The ability to take into account the geographical factor, including call centers of regions and settlements distant from each other in the ERSC, which reduces the risk of critical overloads when the simultaneous occurrence of several emergencies.

As shown in Fig. 2, besides the excess traffic from the CC-Emg, the ERSC can serve the emergency call traffic of its area with intensity $\lambda I(\lambda I-$ intensity of calls arriving), and it is also possible to use the call center (ERSC) resources for outsourcing in the absence of emergency.

In case of emergencies in one or several areas, additional traffic will be arrived on it, excess for these areas. Table 3 presents the results of calculating the required number of workplaces for operators of V(reqERSC) obtained for the given requirements for call losses, namely for $\mathrm{P}$ ERSC $\leq 0.001$ and $\mathrm{P}$ ERSC $\leq 0.01$.

Figure 3 shows the dependence of the number of jobs in the call center ERSC, which need to be highlighted additionally Vadd, on the amount of incoming traffic, expressed through the intensity of the loadAEmg.Also presents the dependence of VreqERSC on the value of the AEmg obtained for the first version of the organization of the ERSC.Both of these dependencies are linear, which reflects the extensive nature of increasing the throughput of the system as a whole. 
Table 3

The results of the calculation of the required number of workplaces of operators in ERSC in case of emergency in one area and the implementation of ERSC based on call center $(\mathrm{CC} \mathrm{j})$

\begin{tabular}{|l|l|l|l|l|l|l|}
\hline \multicolumn{7}{|l|}{ Number of channels in CC $(\mathrm{Vj})=20$ Aj $=10$ Erl } \\
\hline $\begin{array}{l}\text { Incoming } \\
\text { load } \\
\text { inCC-Emg, } \\
\text { AEmg, Erl }\end{array}$ & \multicolumn{2}{|l|}{ PERSC $\leq 0,001$} & \multicolumn{2}{l|}{ PERSC $\leq 0,01$} \\
\cline { 2 - 7 } & $\begin{array}{l}\text { Vreq } \\
\text { ERSC }\end{array}$ & Vadd & Plost & $\begin{array}{l}\text { Vreq } \\
\text { ERSC }\end{array}$ & Vadd & Plost \\
\hline 20 & 26 & 6 & 0.000613 & 22 & 2 & 0.007351 \\
\hline 30 & 37 & 17 & 0.000622 & 32 & 12 & 0.007327 \\
\hline 40 & 48 & 28 & 0.000985 & 43 & 23 & 0.007341 \\
\hline 50 & 60 & 40 & 0.000913 & 54 & 34 & 0.007470 \\
\hline 60 & 72 & 52 & 0.000818 & 64 & 44 & 0.009632 \\
\hline 70 & 83 & 63 & 0.000991 & 75 & 55 & 0.009172 \\
\hline 80 & 95 & 75 & 0.000831 & 86 & 66 & 0.008671 \\
\hline
\end{tabular}

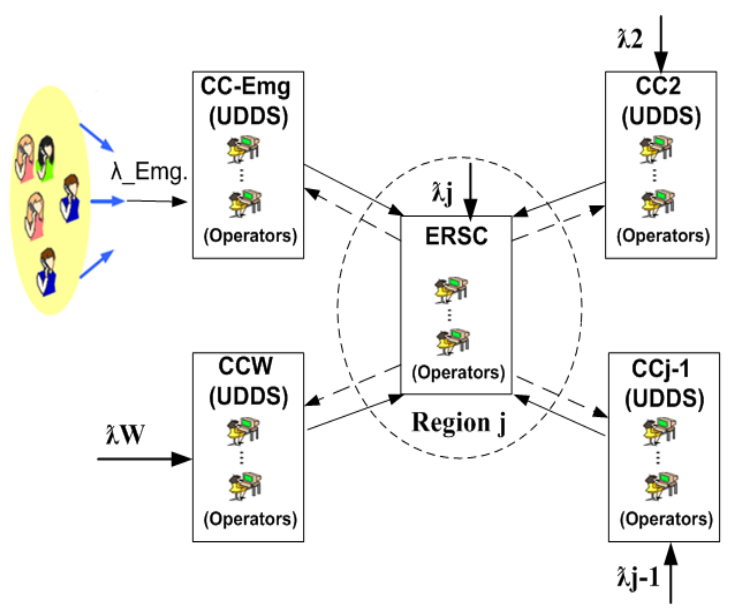

Figure 2. Implementation option of call center ERSC based on call center of the $\mathrm{j}$-th region

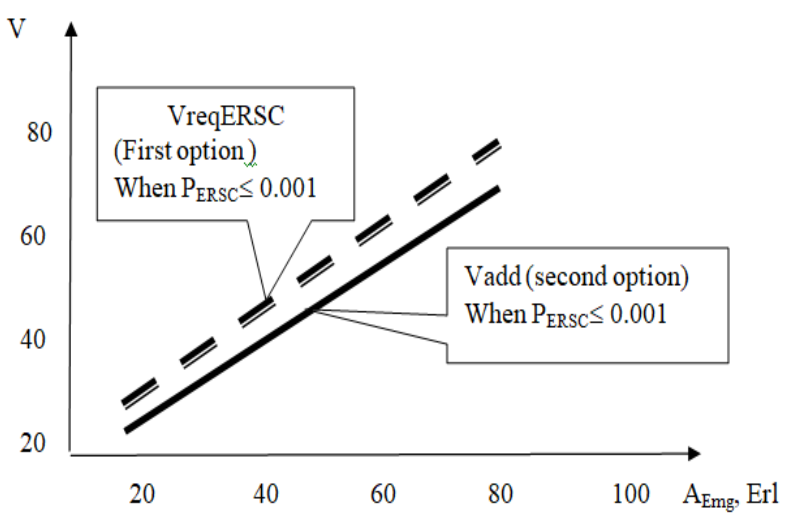

Figure 3. Dependencies Vreq ERSC (first option) and Vadd (second option) on the intensity of the ASF load at RCAF OR $\leq 0.001$

Modern info communication technologies allow expanding the capabilities of communication systems and networks.In particular, we will consider a promising option for organizing mutual assistance by combining the capabilities of emergency call centers in a single re- source. To implement this option in the call centeremergency(ERSC) should be programmatically implemented opportunity of the pseudo-random distribution of emergency calls to call centers of the mutual assistance system taking into account the available resource of operators (see Fig. 4). Practically, we are talking about the implementation of a distributed response center, the resources of which are distributed over separate call service centers of different duty dispatch services.

Describe its functioning, we use the results of calculations by the RDA method.

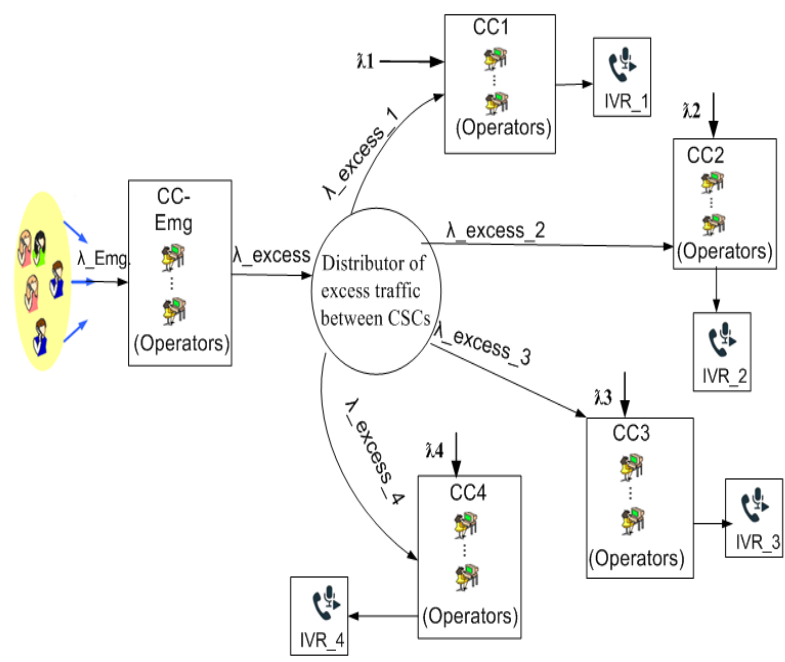

Figure 4. A promising variant of the organization of mutual assistance

When combining several call centers that make up the UDDS into a mutual assistance system, the question arises of organizing an operational reserve in each of the call centers in order to maintain the high quality of service for residents of those service areas at which excess traffic is directed from the emergency call center (ECC).

The requirement must be met - a significant deterioration in the quality of service in the $\mathrm{j}$-th service area is unacceptable if the resource (or part of the resource) of the operator's workplaces in the $\mathrm{j}$-th call center becomes available for excess traffic from the emergency call center.

Practically, talking about that aresource of operator jobs in the call center of the $\mathrm{j}$-th district (operational resource) will be available only to residents of the $\mathrm{j}$-th district (servicing "internal" calls). Only a part of the operator's workplaces in the call center of the j-th district is provided for general needs in a mutual assistance system. Moreover, they can be transmitted on an ongoing basis (they will only serve "external" emergency calls), or can be used both for "external calls" and for "internal calls" of the $\mathrm{j}$-th district.

To investigate the influence of the operational resource on the throughput of the emergency services system in emergency mode, a mathematical model of the functioning of the mutual assistance system is developed.

The calculation results for a mutual assistance system combining four call centers are presented in table 4 . 
During the calculations, it was assumed that an emergency could occur in the area of responsibility of one UDDS (see Fig. 4).Excess traffic from the emergency call center can be directed to the other three call centers of the system $(\mathrm{W}=3)$.It is required to maintain a sufficiently high quality of emergency call service in the area of responsibility of those EDDS where there is no emergency, but where excess traffic is redirected from the emergency zone.To this end, it is proposed to allocate part of the resource of operator systems for internal needs, providing as a general emergency resource only part of the available funds.In table 4 , the number of operator positions allocated to the operational reserve is denoted by Vjrez, and varied from 0 to 6 (up to $30 \%$ of the available resource).

As shown in Fig. 5, the dependences of Pivr (the proportion of the excess traffic directed to the IVR)) and Pivrj (the proportion ofthe trafficarriving in the zone $\mathrm{j}$ directed to the IVR) on the value of the operational reserve are multidirectional, namely, an increase in Vjrez reduces the efficiency of the entire system (the percentage of failures in excess emergency traffic increases), but a high level of emergency call service remains in areas outside to the emergency zone.

Table 4

Results of calculating the probability of direction calls from the emergency area Pivr Emg to IVR, and the probability of direction calls from the $\mathrm{j}$-th service area to IVR depending on the operational reserve Vjrez

\begin{tabular}{|c|c|c|}
\hline \multicolumn{3}{|c|}{$\begin{array}{l}\text { Initial data: } V j=V E m g=20, j=1, \ldots, W \\
A j=10 \mathrm{Erl} \quad \mathrm{W}=3\end{array}$} \\
\hline \multicolumn{3}{|c|}{$\mathrm{A} \mathrm{Emg}=20 \mathrm{Erl}$} \\
\hline Vjrez & Pivr Emg & Pivrj \\
\hline 0 & 0.00487 & 0.00487 \\
\hline 1 & 0.01322 & 0.00440 \\
\hline 2 & 0.02712 & 0.00399 \\
\hline 3 & 0.049328 & 0,00361 \\
\hline 4 & 0.08302 & 0,00328 \\
\hline 5 & 0.13138 & 0,00299 \\
\hline 6 & 0.19684 & 0,00274 \\
\hline \multicolumn{3}{|c|}{ A Emg $=40$ Erl } \\
\hline 0 & 0,08475 & 0,08475 \\
\hline 1 & 0,15540 & 0,05180 \\
\hline 2 & 0,22194 & 0,03264 \\
\hline 3 & 0,28893 & 0,02118 \\
\hline 4 & 0,35821 & 0,01418 \\
\hline 5 & 0,43009 & 0,00980 \\
\hline
\end{tabular}

We fix the requirements for the quality of emergency call service in the mutual assistance system at a high level. Namely, we will set the requirement for the probability of failures for calls from the emergency zone at the level PivrEmg $\leq 0.01$. Table 5 presents the results of calculating the maximum allowable value of the operational reserve Vjrez max.Part of the emergency traffic is served in the emergency call center (CC-Emg), and some of it with intensity exec is directed to other CCs of mutual assistance system. If the condition Vjrez $\leq$ Vjrez max is not satisfied, then, accordingly, the set PivrEmg $\leq 0.01$ requirement will not be fulfilled.
The value of AEmgalow - this is the permissible load on the call-emergency, the excess of which does not allow to fulfill the requirement PivrEmg $\leq 0.01$.

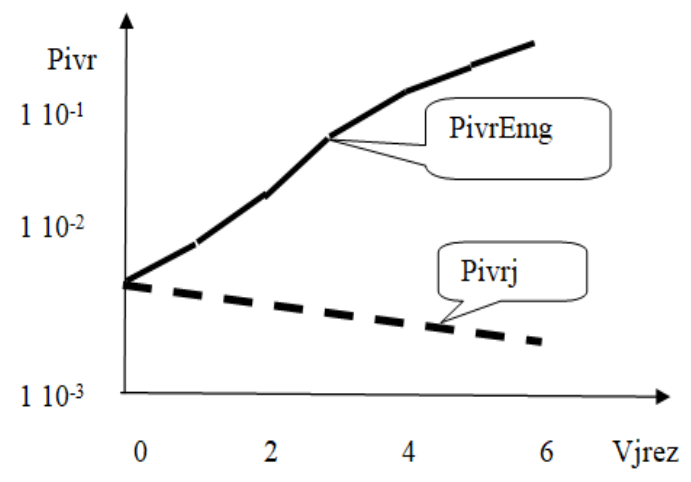

Figure 5. Dependencies PivrEmg and Pivrj on the value of the operational reserve Vjres

Analyzing the data of table 5, we can conclude that the allocation of a large operational reserve in the call center (CC) of the system limits the effect of mutual assistance in emergency mode.For example, if in a call center with a capacity of $\mathrm{Vj}=30$, for the operational reserve will be left $\mathrm{Vj}$ res $=10$ operator jobs at $\mathrm{Aj}=10$ Earl, then in the $\mathrm{j}$-th area, almost trouble-free service of emergency calls will be implemented, and for calls from the emergency zone, the requirement is Pivr Emg $\leq 0.01$ will only be executed if the AEmg does not exceed 29.38 Erl. Otherwise, we can expect a sharp increase in the value of PivrEmg.

Table 5

The results of calculating the probabilities of PivrEmg and Pivrj at $\mathrm{W}=3$ of the value of the operational reserve Vjmax, if the requirement is PivrEmg $\leq 0.01$ and at $\mathrm{Aj}=10 \mathrm{Erl}$

\begin{tabular}{|l|l|l|l|l|}
\hline \multicolumn{6}{|c|}{ V_Emg=Vj Aj=10 Erl W=3 } \\
\hline Vj & Vjrez max & AEmg max, Erl & Pivr Emg & Pivr j \\
\hline 20 & 1 & 18,14 & 0.009934 & 0.003311 \\
\hline 24 & 4 & 24,18 & 0.009990 & 0.002145 \\
\hline 30 & 10 & 29,38 & 0.00998 & $2.21^{*} E-8$ \\
\hline 36 & 16 & 34,71 & 0.009968 & $3.5^{*} E-10$ \\
\hline
\end{tabular}

We will make a demand for emergency calls service in areas where there are no emergencies, to the call service centers of which the excess traffic is routed Pivrj $\leq 0.01$, setting the requirement PivrEmg $\leq 0.01$ for calls from the emergency zone.

The purpose of the calculation is to clarify the value of the permissible intensity of excess traffic A_excess, as well as the permissible value of the traffic intensity in the emergency call center AEmg_allow. The calculation results are presented in table 6 and Fig. 6. 
Table 6

The results of calculating the permissible excess load A excess depending on the maximum number of channels of the operational reserve Vjrez with the initial data: $\mathrm{Vj}=30 \mathrm{~W}=3$ and $\mathrm{Aj}=10$ Earl; PivrEmg $\leq 0.01$ and $\operatorname{Pivrj} \leq 0.01$

\begin{tabular}{|l|l|l|l|l|}
\hline \multicolumn{5}{|l|}{ Vj=30 W=3 } \\
\hline Vjrez Max & AEmg_allow & A_excess & PivrEmg & Pivrj \\
\hline 0 & 60.01 & 30,90 & 0.009964 & 0.009964 \\
\hline 1 & 55.78 & 26,79 & 0.009964 & 0.002491 \\
\hline 2 & 52.69 & 24,07 & 0.009974 & 0.000785 \\
\hline 3 & 49.91 & 21,15 & 0.00999 & 0.000269 \\
\hline 4 & 47.18 & 18,57 & 0.009996 & $9.721^{*} \mathrm{E}-5$ \\
\hline 5 & 44.45 & 16,02 & 0.00998 & $3.645^{*} \mathrm{E}-5$ \\
\hline 6 & 41.71 & 13,51 & 0.00999 & $1.424^{*} \mathrm{E}-5$ \\
\hline 7 & 38.88 & 10,98 & 0.00995 & $5.762^{*} \mathrm{E}-6$ \\
\hline 8 & 35.97 & 8,49 & 0.00995 & $2.43{ }^{*} \mathrm{E}-6$ \\
\hline 9 & 32.89 & 6,03 & 0.00997 & $6.91{ }^{*} \mathrm{E}-8$ \\
\hline 10 & 29,38 & 3,57 & 0.00998 & $2.21{ }^{*} \mathrm{E}-8$ \\
\hline
\end{tabular}

The analysis of the obtained dependence shows that, subject to the set requirements for the values of PivrEmg and Pivrj, the value of AEmg_allow and the value of the operational reserve Vjrez max are interrelated.

For this variant, it may be recommended to limit Vjrez max to Vjrez max $=4$.If we choose an operational reserve of $\mathrm{Vjrez}=5$ or more, then we get almost troublefree emergency call service in the $\mathrm{j}$-th area $(\mathrm{CCj}$ is not loaded), and the coefficient of potential stability of the CC-Emg to overloads $h$ does not rise above the value $h=$ 4.718 at Vjrez $=$ Vjrezmax $=4$.It should be noted that the inclusion in the mutual assistance system of 26 call center operators of the $\mathrm{j}$-th service zone out of thirty means that all thirty center operators will also be available to residents of the $\mathrm{j}$-th service zone, and in addition, 26 operators will be included in the mutual assistance system in case of emergency .

Part of the available resource of operators on call centers should remain in the operational reserve. The value of $V_{i}^{m a}$ can also be used, which characterizes the number of jobs in $\mathrm{CCj}$ included in the mutual assistance system minus the operational reserve.

We present in table 7 the option of resources distribution to individual call centers of the system, converting the data of table 1.Required aggregate resource of operator jobs to service excess traffic in the system and was defined as V_system = Vreq-emg and was performed for two versions of the given requirements of the $P_{\text {_ system }} \leq 0.001$ and the $P$ _system $\leq 0.01$ (where $\mathrm{P}_{-}$system - the probability of failure of excess traffic in the system).Next, the number of operators included in the mutual assistance system at each of the call centers was determined.

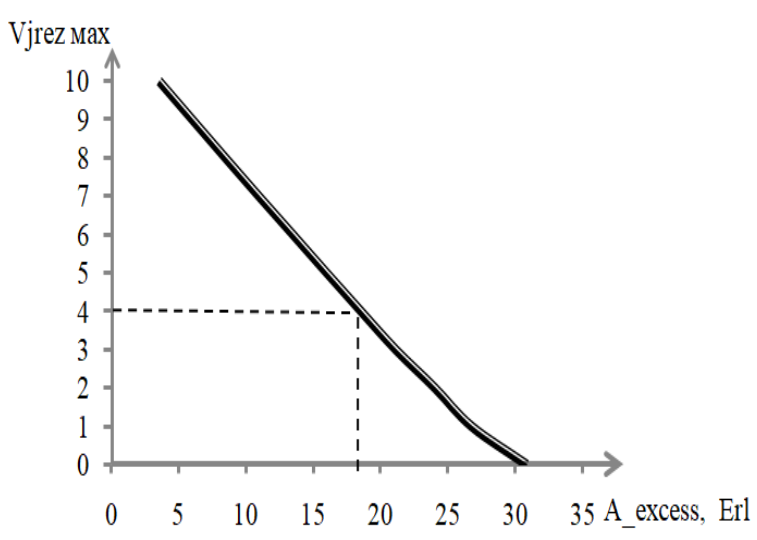

Figure 6. Dependence of the maximum allowable number of channels of the operational reserve on the permissible excess load at $\mathrm{Vj}=30, \mathrm{~W}=3, \mathrm{Aj}=10 \mathrm{Erl}$

In this case

Vreq-emg $=V_{1}^{m a}+V_{2}^{m a}+V_{3}^{m a}$.

Accordingly, the operational reserve in each of the call centers of the system is

$$
\text { Vjrez }=\mathrm{Vj}-\mathrm{V}_{\mathrm{i}}^{\mathrm{ma}} \text {, }
$$

Where $V_{i}^{m a}$ - number of channels dedicated to serving excess traffic.

It was assumed that the average level of traffic in the area is at 10 Erl. In fact, the case of a fivefold increase in traffic in the emergency area to the ASF $=50 \mathrm{Erl}$ was considered.

Analyzing the result, we can conclude that the system will provide a quality of service of the $\mathrm{P}$ system $\leq$ 0.01 with a fivefold increase in traffic if $4-5$ operator jobs are allocated as an operational reserve in each call center

Table 7

Results of calculating the required number of operator jobs in the mutual assistance system in case of emergencies in one area. The number of channels in $\mathrm{CC}-\mathrm{EmgVcc}-\mathrm{Emg}=20, \mathrm{~V} 1=\mathrm{V} 2=\mathrm{V} 3=20$

\begin{tabular}{|c|c|c|c|c|c|c|c|c|}
\hline \multirow{2}{*}{$\begin{array}{l}\text { Incoming } \\
\text { load } \\
\text { in CC-Emg, } \\
\text { AEmg, Erl }\end{array}$} & \multicolumn{4}{|c|}{ Psystem $\leq 0,001$} & \multicolumn{4}{|c|}{ P system $\leq 0,01$} \\
\hline & $\begin{array}{l}\text { Vreq- } \\
\text { emg }\end{array}$ & $V_{1}^{m a}$ & $V_{2}^{m a}$ & $V_{3}^{m a}$ & $\begin{array}{l}\text { Vreq- } \\
\text { emg }\end{array}$ & $V_{1}^{m a}$ & $V_{2}^{m a}$ & $V_{3}^{m a}$ \\
\hline 20 & 18 & 6 & 6 & 6 & 14 & 5 & 5 & 4 \\
\hline 30 & 29 & 9 & 10 & 10 & 24 & 8 & 8 & 8 \\
\hline 40 & 41 & 14 & 14 & 14 & 35 & 12 & 12 & 11 \\
\hline 50 & 53 & 18 & 18 & 17 & 46 & 16 & 15 & 15 \\
\hline
\end{tabular}

The implementation of the presented variant of the organization of mutual assistance involves the presence of two modes of operation of the system.

1. In the absence of an emergency, call service centers within the UDDS, combined into the system, operate independently of each other. 
2. When the emergency mode is fixed, excess traffic is directed from the emergency call center (CC-Emg) to the other call centers (CC) of the system, being distributed in a pseudo-random manner, and taking into account the available operator capacity in each call center.

Note the special requirements:

- It is necessary to quickly enough determine the onset of emergency, allowing you to switch to the second mode of operationin the CC-Emg. There should be information about available operators in other call centers included in the mutual assistance system;

- Since it is not known in which region the emergency will occur, such information should be in each call center of the system;

- It is also necessary to provide protection against possible collisions of the simultaneous occupation of the same resources, the occurrence of which is possible with a significant removal of call centers from each other.

Commercial emergency call centers with a high technical level can be included in the mutual assistance system of emergency services. As the experience of work of call centers of various telecom operators shows, exceeding the value of using the operator's workplace $\mathrm{U}>0.6$ increases staff turnover and staff retraining costs.Let us calculate the AEmg_allow because the value $\mathrm{Aj}$ is fixed at the level $\mathrm{Aj}=\mathrm{U} * \overline{\mathrm{V}} \mathrm{j}=0.6 * \mathrm{Vj}$.

The results of calculating the coefficient of potential stability of the CC-Emg for overloads $\mathrm{h}$ at $\mathrm{P}$ system $\leq$ 0.01 are presented in table 8.Analyzing the results, we can conclude/

The degree of influence of the value of $\mathrm{W}$ in the mutual assistance system on the coefficient $h$ decreases when the requirement $\mathrm{Aj}=0.6 * \mathrm{Vj}$ is met. For example, at $\mathrm{Vj}=40$, an increase in $\mathrm{W}$ from unity to four ensures a 1.4 -fold increase in the coefficient $h$. In the presence of four combined call centers $(\mathrm{W}=3)$, the choice of $\mathrm{Vj}=30$ ensures stability $\mathrm{h}=2.05$.

Table 8

The results of calculating the permissible load intensity of AEmg allow, at which the conditions of the P_system $\leq 0.01$ will be satisfied at $\mathrm{Aj}=0.6 * \mathrm{Vj}$. Erl

\begin{tabular}{|l|l|l|l|l|l|l|l|}
\hline $\begin{array}{l}\text { Number of } \\
\text { operators, } \\
\text { Vj }\end{array}$ & Aj & $\begin{array}{l}\text { AEmg } \\
\text { allow } \\
\text { Erl }\end{array}$ & $\begin{array}{l}\text { Stability } \\
\text { coefficient } \\
\mathrm{h}\end{array}$ & $\begin{array}{l}\text { Number of } \\
\text { operators, } \\
\text { Vj }\end{array}$ & Aj & $\begin{array}{l}\text { AEmg } \\
\text { allow } \\
\text { Erl }\end{array}$ & $\begin{array}{l}\text { Stability } \\
\text { coefficient } \\
\text { h }\end{array}$ \\
\hline $\mathrm{W}=1$ & \multicolumn{7}{|l|}{$\mathrm{W}=2$} \\
\hline 24 & 14.4 & 18.85 & 1.31 & 24 & 14.4 & 21.01 & 1.46 \\
\hline 30 & 18 & 27.24 & 1.51 & 30 & 18 & 31.02 & 1.72 \\
\hline 36 & 21.6 & 35.24 & 1.63 & 36 & 21.6 & 40.67 & 1.88 \\
\hline 40 & 24 & 40.54 & 1.69 & 40 & 24 & 47.09 & 1.96 \\
\hline $\mathrm{W}=3$ & 14.4 & 22.66 & 1.57 & 24 & 14.4 & 24.07 & 1.67 \\
\hline 24 & 18 & 34.13 & 1.89 & 30 & 18 & 36.96 & 2.05 \\
\hline 30 & 21.6 & 45.33 & 2.10 & 36 & 21.6 & 49.7 & 2.3 \\
\hline 36 & 24 & 52.84 & 2.2 & 40 & 24 & 58.28 & 2.43 \\
\hline 40 &
\end{tabular}


The simplicity of its implementation has several advantages: in the work of the distributor, one can take into account such a factor as combining call centers of different capacities into a mutual assistance system (a possible option in the Republic of Yemen).

Operational redistribution of traffic flows during overload or failure of individual emergency call centers is possible.

Mutual assistance can be implemented on an ongoing basis:

- At the third stage, it is necessary to analyze the statistics on the increase in the number of emergency calls in emergency mode, specifying the requirements for the value of $\mathrm{h}$ - the coefficient of potential resistance of the $\mathrm{CC}$-Emg to overloads. Further, taking into account the value of $h$, the capacity of the operator services and the number of emergency call centers to be included in the mutual assistance system should be determined.

- The fourth stage involves the selection of a specific option for the operational reservation of operator system resources to maintain an acceptable level of emergency call service in the areas of responsibility of the UDDS, which are part of the mutual assistance system, but are not covered by emergencies.

- The five stages involves the choice of variant of building an emergency call service system, taking into account the results of calculating the system work indicators, subject to the general requirement of minimizing costs and efficient use of resources (including involving the resources of a commercial call center).

\section{CONCLUSION}

1. Research and analytical calculation of the characteristics of the mutual assistance system of emergency services allowed to determine the positive and negative sides of each of the options considered.

2. The stages of combining and efficient use of resources of the emergency call service system are determined.

3. Clarification of the requirements for the value of $\mathrm{h}$ - the coefficient of potential stability - a necessary condition for choosing the option of building a mutual assistance system.

4. Formation of an operational reserve of operator jobs in an emergency call center allows maintaining a high level of service quality, but reduces the effectiveness of the mutual assistance system as a whole.

\section{REFERENCES}

1. Mohammed O.A.A. Development of approaches to ensure reliable emergency communications in emergencies. T-Comm. 2020. Vol. 14. No.1. P. 42-48. (in Russian)

2. Stepanova I.V. Use of flexible routing corporate callcenters. T-Comm. 2015. Vol. 9. No.11. P. 25-31. (in Russian)

3. Pshenichnikov A.P. Theory of Teletraffic. Textbook for high schools. Moscow: Hot line - Telecom, 2017. 212 p.

4. Stepanov S.N. Theory of teletraffic: concepts, models and applications. Moscow: Hot line - Telecom, 2015. 868 p.

5. Stepanova I.V., Ahmed Abdulvasea, Ndayinkunda Zhuven. Analysis of promising approaches to improve the reliability of converged enterprisenetworks. T-Comm. 2015. Vol 9. No.12. P. 44-51. (in Russian) 